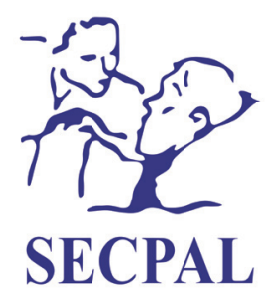

Medicina Paliativa

www.medicinapaliativa.es

ORIGINAL

\title{
Barreras y recursos para cuidar: un abordaje cualitativo fenomenológico de la realidad del cuidador formal
}

\author{
Paula Hidalgo-Andrade* y Silvia Martínez-Rodríguez
}

Universidad de Deusto, Facultad de Psicología y Educación, Bilbao, España

Recibido el 2 de marzo de 2018

Aceptado el 16 de abril de 2018

\begin{abstract}
PALABRAS CLAVE
Cuidador,

satisfacción personal, autocuidado, política pública, investigación cualitativa, Ecuador.
\end{abstract}

\begin{abstract}
Resumen
Objetivo: Este estudio propone explorar la realidad de los cuidadores formales de personas con enfermedades crónicas, degenerativas y en cuidados paliativos, analizando las barreras y recursos que ellos perciben para realizar su labor.

Método: Entre octubre y diciembre de 2016, 84 cuidadores formales de tres instituciones privadas de varios niveles de atención en Quito (Ecuador) participaron en entrevistas semiestructuradas que fueron grabadas en audio, transcritas verbatim y analizadas desde el enfoque fenomenológico utilizando el software Atlas.ti 7.5.10.

Resultados: Las barreras estatales referentes a la falta de provisión de servicios por parte del Estado fueron las más prevalentes. Por otro lado, la satisfacción y el sentido de servir y cuidar fueron los recursos más utilizados. Las otras situaciones identificadas se ubicaron en un espectro entre barreras y recursos, dependiendo de cómo se las manejara. Estas fueron el conocimiento y la comprensión de las enfermedades y los cuidados paliativos, el apoyo social, las vivencias personales y el autocuidado.

Conclusiones: Estos cuidadores formales perciben gran satisfacción en su labor; sin embargo, también se enfrentan diariamente a situaciones que pueden ser percibidas como barreras para cuidar. Los costes y beneficios que experimentan durante sus actividades diarias influyen directamente en la percepción de su labor, en la calidad de cuidado que brindan y en la calidad y cantidad de autocuidado que practican. Comprender y gestionar efectivamente estas vivencias puede apoyar positivamente al cuidador formal.
\end{abstract}

\footnotetext{
*Autor para correspondencia:

Paula Hidalgo-Andrade

Facultad de Psicología y Educación. Universidad de Deusto. Avda. Universidades, n. ํ24, 48007 Bilbao.

Correo electrónico: paula.hidalgo@udla.edu.ec
} 


\section{KEYWORDS}

Caregiver, personal

satisfaction, self-

care, policy, qualitative research, Ecuador.

\begin{abstract}
Objective: This study explored the reality of formal caregivers for people in palliative care and with chronic or degenerative diseases by analyzing the barriers and resources they face. Method: Between October and December 2016, 84 formal caregivers from three private institutions of various levels of care in Quito (Ecuador) participated in semi-structured interviews that were recorded on audio, transcribed verbatim, and analyzed from a phenomenological perspective using the Atlas.ti 7.5.10 software package.

Results: External barriers regarding the lack of provision of education and public services by the State were most prevalent. On the other hand, the most predominant resources were satisfaction and sense of meaning in serving and caring. Other aspects lied along the continuum between barriers and resources, depending on how they were handled. These included: knowledge and understanding of illnesses and palliative care, social support, personal experiences, and self-care. Conclusions: Formal caregivers for people with chronic, degenerative, and palliative care diseases perceive great satisfaction in their work; however, they also face daily situations that may be perceived as barriers to caring. The costs and benefits experienced during their daily activities directly influence the perception of their work, the quality of care they provide, and the quality and quantity of self-care they practice. Understanding and effectively managing these experiences may positively support formal caregivers.
\end{abstract}

Hidalgo-Andrade P, Martínez-Rodríguez S. Barreras y recursos para cuidar: un abordaje cualitativo fenomenológico a la realidad del cuidador formal. Med Paliat. 2019;26(2):113-119.

\section{Introducción}

Más de 20 millones de personas en el mundo necesitan cuidados paliativos anualmente. De este total, aproximadamente un tercio tiene cáncer y el resto presenta enfermedades degenerativas y crónicas o potencialmente mortales ${ }^{1}$. Estas cifras incluyen únicamente a la persona con enfermedad y no reflejan el apoyo que también se brinda a los familiares. Los cuidadores formales ofrecen tal apoyo, y son aquellas personas asociadas con un sistema de servicio formal, sea como voluntarios o como trabajadores remunerados ${ }^{2}$. A pesar de la amplia demanda de cuidados paliativos, solo 20 países los tienen integrados apropiadamente en sus sistemas de atención sanitaria ${ }^{1}$; hasta la actualidad, Ecuador no es uno de ellos ${ }^{3}$.

Cuidar a personas en estas situaciones muestra a los cuidadores "su propia vulnerabilidad como ser humano, recordándole su propia muerte y lo que podría percibirse como más cruel: la posibilidad de una 'muerte en vida'"'4. Asimismo, el contacto continuo con la realidad inmodificable de los pacientes, familiares y su entorno, la eventual muerte de la persona, el proceso de duelo y la presión por parte de pacientes y familiares ${ }^{5}$ pueden hacer que los cuidadores tiendan a dejar de lado su propio cuidado para enfocarse en las necesidades de los demás ${ }^{6}$.

Esta gran carga emocional y psicológica coloca al cuidador formal en riesgo de desarrollar burnout y fatiga de compasión, definida como "el coste de cuidar a alguien que sufre", aspectos asociados con repercusiones negativas en la salud del cuidador y con el desgaste de la relación interpersonal entre el cuidador y el paciente ${ }^{7}$, que también pueden influir negativamente en el ajuste del paciente con los cambios que se dan durante el progreso de su enfermedad ${ }^{8}$. Igualmente, se relacionan con baja productividad, baja motivación para cuidar y problemas en las propias relaciones interpersonales del cuidador?.

Existen inconsistencias en los niveles reportados de estrés, burnout y desgaste por empatía en cuidadores formales ${ }^{10,11}$. Esta variación en la prevalencia se da por la diversidad de medidas utilizadas, la baja calidad de la metodología en los estudios y la falta de claridad en los constructos ${ }^{12}$. Por otro lado, el trabajar ayudando a personas que necesitan cuidados genera satisfacción o placer ${ }^{13}$. Estos se dan a través de relaciones significativas con uno mismo, el paciente, sus familiares y la comunidad en general ${ }^{7}$. Los cuidadores con mayor satisfacción experimentan más afectos positivos, se esfuerzan y dedican más, sienten energía y orgullo, perciben su trabajo con propósito, presentan un mayor compromiso, se comunican mejor, brindan información apropiada a los pacientes y sienten mayor eficacia y seguridad en la relación interpersonal con ellos ${ }^{14}$.

\section{Objetivo de la investigación}

Debido a la compleja realidad de la labor de cuidar, se deben explorar los factores que contribuyen al desarrollo de las consecuencias negativas del cuidado y reconocer y potenciar la satisfacción y autocuidado para evitar o mitigar el posible desgaste del cuidador formal. Para lograrlo, es importante conocer la manera en que ellos perciben su realidad. El propósito de este estudio cualitativo basado en la fenomenología fue explorar las barreras y recursos que los cuidadores formales de personas con enfermedades crónicas, degenerativas y en cuidados paliativos experimentan 
para realizar su labor. Esta investigación tuvo la aprobación del Comité de Ética de la Universidad de Deusto y reporta la primera parte de un estudio longitudinal de tipo mixto cuyos objetivos eran explorar esta realidad y desarrollar, implementar y analizar los resultados a largo plazo de una intervención grupal para aumentar la satisfacción y el autocuidado de los cuidadores formales.

\section{Metodología}

\section{Diseño}

Se realizaron entrevistas individuales semiestructuradas para poder capturar aspectos de la realidad del cuidador que las medidas cuantitativas no logran ${ }^{15}$. La metodología cualitativa empleada permitió explorar experiencias individuales en las situaciones y contextos en las que estas sucedían ${ }^{16}$. Los datos se analizaron con el enfoque fenomenológico para "entender los fenómenos sociales desde la propia perspectiva del actor" 17 y comprender profundamente la realidad del cuidador formal.

Una vez reclutados los participantes, se coordinaron las citas con cada uno mediante una llamada telefónica. Las entrevistas fueron llevadas a cabo por la investigadora principal, fueron grabadas en audio y tuvieron lugar en las respectivas instituciones, a excepción de cinco que se llevaron a cabo en los domicilios de las participantes por su conveniencia logística. Las entrevistas tuvieron lugar entre octubre y diciembre de 2016. Su duración media fue de 25 minutos e incluían preguntas como “¿qué te motivó a ser cuidador?”, “¿qué crees que se necesita para este tipo de trabajo?”, “¿hay alguna barrera que se interponga para realizar tu labor?”, “¿qué recursos crees tener para realizar mejor tu trabajo?”, y “ ¿te has sentido agotado al realizar tu labor?" Durante las entrevistas no se tomó ningún tipo de apuntes para no interrumpir su fluidez. Para asegurar la confidencialidad de los participantes, las transcripciones fueron realizadas por la misma entrevistadora; estas no fueron devueltas a los participantes.

Para garantizar la calidad de la evaluación científica se utilizó la guía para publicar artículos cualitativos ${ }^{18}$ y los cuatro criterios de Guba y Lincoln ${ }^{19}$. La credibilidad se avaló durante el transcurso de las entrevistas mediante la retroalimentación con los participantes para asegurar la comprensión correcta de la información que otorgaban; además, se tomaron notas de campo después de cada entrevista para mantener un registro de situaciones no verbales. Para asegurar la conformabilidad todas las entrevistas se llevaron a cabo con un guion, fueron grabadas, se transcribieron verbatim y se debatieron las categorías encontradas con un segundo investigador, garantizando así también la dependencia interna. La dependencia externa se aseguró explicando claramente los criterios de inclusión de la muestra y los contextos donde se llevaron a cabo las entrevistas. Finalmente, se señala la limitación en la transferibilidad de los datos debido al contexto cultural y geográfico de los entrevistados.

\section{Participantes}

Los participantes fueron reclutados por medio de reuniones presenciales mantenidas en instituciones de Quito,
Ecuador. Participaron tres instituciones privadas: una fundación con centro de día para personas con Alzheimer y otras demencias, un hospice especializado en cuidados paliativos que cuenta también con un servicio de visitas domiciliarias, y el equipo paliativo y de emergencia de un hospital oncológico. En las reuniones se explicaron las características y propósito del estudio; las personas interesadas firmaron el consentimiento informado que indicaba la confidencialidad y voluntariedad de su participación, así como los datos de contacto de la investigadora principal. Las 100 personas que aceptaron participar fueron entrevistadas.

Debido al propósito de la investigación, se excluyeron de la muestra las entrevistas de aquellos cuidadores que no mantenían una relación directa con pacientes o sus familiares. La muestra final se compuso de 84 entrevistas, 11 hombres y 73 mujeres. Los participantes fueron 23 enfermeras, 20 voluntarios, 19 auxiliares de enfermería, 15 médicos, 3 psicólogos, 3 terapeutas ocupacionales y 1 trabajador social. La edad media de los participantes fue de 42,4 años $(D E=13,59)$, con un rango de 22 a 70 años, y el tiempo promedio como cuidadores formales fue de 7,38 años $(D E=6,22)$, con un rango de 10 meses a 24 años. Los participantes no recibieron ningún tipo de compensación económica por colaborar en el estudio.

\section{Análisis de datos}

Se codificaron y analizaron las transcripciones de las entrevistas con el software Atlas.ti 7.5.10. Los códigos fueron estratificados en temas y subtemas para identificar posibles categorías.

\section{Resultados}

En esta investigación las barreras se contextualizaron como las situaciones que el cuidador percibe como limitantes para realizar su labor, mientras que los recursos como aquellos apoyos o motivaciones para continuar. Los resultados concuerdan con otros estudios en que las circunstancias familiares y personales son más prevalentes que las relacionadas directamente al cuidado ${ }^{16}$. Además, recalcan la importancia de las políticas estatales que influyen directa e indirectamente en la calidad de cuidado que se brinda.

Las entrevistas resaltaron seis temas principales: (i) aptitudes, conocimiento y comprensión sobre las enfermedades y los cuidados paliativos; (ii) apoyo social y balance de vida personal; (iii) experiencias personales; (iv) autocuidado; (v) satisfacción y sentido, y (vi) barreras externas de política pública.

Aptitudes (80/84), conocimiento y comprensión sobre las enfermedades y los cuidados paliativos (81/84). Uno de los recursos imprescindibles para realizar su labor es tener conocimiento específico sobre las enfermedades y los cuidados paliativos. Además, se deben tener y cultivar aptitudes como determinación, alegría, empatía, paciencia, perseverancia, sensibilidad, tranquilidad, humanidad, humor, deseo de servicio y reconocimiento de limitaciones personales.

El desconocimiento y la falta de comprensión sobre las demandas de las enfermedades y el verdadero propósito de los cuidados paliativos es uno de los impedimentos prin- 
cipales del cuidador formal, porque esta ignorancia puede conducir a miedos y prejuicios. Además, hace que las personas no acudan a este tipo de cuidados oportunamente. Ante esto se sugiere educar a la población aunque "se les trata de informar a las personas, pero muchas veces se resisten a aceptar la enfermedad... [a aceptar] que sus seres queridos están llegando a una terminalidad de sus vidas (...)." (Trabajadora social).

"Otro problema son los médicos. Los médicos que son formados para curar y que no saben decir hasta aquí y esta persona tiene que ir a cuidados paliativos..., que luchan y luchan hasta el final, muchas veces a costos altos de la familia y del paciente $\mathrm{y}$, sobre todo, a costo del sufrimiento de las persona" (Enfermera). En ocasiones también se percibía que los cuidadores familiares ponían una barrera, especialmente cuando su agotamiento era evidente: "[Los familiares] están súper cansados y ya no ven ninguna salida. Cuando el cuidador está muy negativo, eso a mí me hace perder la paciencia y se me hace difícil conectarme porque en verdad empiezo a tener una resistencia hacia este cuidador" (Psicóloga).

Según las personas entrevistadas, la población en general y los propios cuidadores sanitarios tienen una idea poco clara de las enfermedades y los cuidados paliativos. En otros contextos culturales también se ha encontrado que los médicos limitan su concepto de cuidados paliativos al manejo de dolor y síntomas, desconocimiento que podía atrasar la provisión de los cuidados paliativos necesarios ${ }^{20}$.

Apoyo social (74/84) y balance con la vida personal (40/84). Dentro de cada institución, los entrevistados indicaron que el contar con los compañeros y supervisores para compartir dudas y emociones les ayuda a trabajar mejor para el bien del paciente y su familia: "Tener ese espacio creo que sí ha sido bueno porque se siente el respaldo y se puede hacer cosas para cambiar estas cuestiones que son netamente institucionales, y entender que habrá cosas que sí puedo cambiar con ayuda y habrá otras cosas que no." (Psicóloga). De igual manera, contar con el apoyo de su propia familia ayuda a mantener un sano distanciamiento entre su rol de cuidador y su vida personal: "El poder compartir un poquito de lo que hago aquí en mi casa, para poder olvidar lo que hago aquí y poder dedicarme a mi casa..., esa puede ser mi herramienta" (Enfermera).

El apoyo social beneficia al cuidador; sin embargo, en ocasiones es difícil mantener o cultivar esta ayuda porque la economía y los horarios implican menor tiempo para compartir con otras personas y actividades fuera del rol de cuidador. "Este es un trabajo que te da razones para vivir, pero no te da para vivir. Significa que la mayoría tenemos que tener un trabajo más que sí nos dé para vivir, para poder hacer este. Y eso significa doble trabajo, significa que debes dividir tu tiempo, que tienes que hacer todos los arreglos necesarios para dividirte un poquito tú también" (Médica voluntaria).

Experiencias personales (50/84). El haber vivido situaciones personales o familiares de enfermedad y sufrimiento ayuda a desarrollar una mayor empatía en los cuidadores formales. "El tener ese doble rol, eres enfermo y el que cuida al enfermo, te hace entenderles con mayor facilidad" (Médico). Igualmente, las vivencias como cuidador en este tipo de cuidado enfrentan a los cuidadores a la finitud de su propia existencia: "Cuando uno va cumpliendo años, no es que se tenga miedo a la muerte... Porque uno está preparado, esto te hace preparar más inclusive, pero cada vez la expectativa de vida es menos..." (Voluntaria).

Además, el vínculo que se genera entre los pacientes y cuidadores durante el tiempo de cuidado puede ser difícil de gestionar; sobre todo cuando la realidad del paciente revive experiencias de enfermedad y sufrimiento del propio cuidador. "Mi madre murió con cáncer [llora] (...). Esa ha sido mi barrera y tal vez no he podido vencer esa etapa de sobrellevar y tal vez un sentimiento de culpa de no haber hecho más por mi madre" (Enfermera).

De manera similar, el cuidado pediátrico parece despertar emociones que influyen en los cuidadores formales. Algunos estudios han indicado que muchos no están formados adecuadamente para brindar este tipo de cuidados a niños y sus familiares ${ }^{21}$. Del mismo modo, para algunos médicos no está claro el momento apropiado para introducir los cuidados paliativos para niños y adolescentes y no se sienten cómodos con el término "cuidados paliativos" en esta población ${ }^{20}$. En estas entrevistas, la atención pediátrica no fue una barrera directa para realizar su labor sino un detonante para experiencias emocionales profundas que pueden convertirse en barreras. "Ese joven me hizo pedazos... Me deprimió... Que lloraba... Le tenía aquí [señala la garganta], le tenía atragantado al joven. Falleció el joven. Desde ahí creo que puse un poquitito más de barrera al apegarme mucho con la mamá, el papá, a la familia en sí... A él mismo" (Enfermera). Los participantes que mencionaron el cuidado a niños le atribuyeron a este una carga emocional mayor. Además, aquellos que también son padres indicaron una preocupación adicional ante la posibilidad de que algo similar les sucediese a sus propios hijos.

Otras preocupaciones cotidianas fuera de su rol de cuidadores también surgieron en las entrevistas como posibles detonantes de fatiga en su trabajo. Una participante lo dijo claramente: "Cansada y agotada por la labor en la institución, no; [me he sentido] cansada y agotada porque tengo una carga emocional personal..." (Médica voluntaria).

Autocuidado (36/84). Además de las medidas de bioseguridad, los participantes identificaron el autocuidado como algo que haces para ti y para los tuyos con el objetivo de "desconectarse" y seguir. Entre las técnicas de autocuidado utilizadas se mencionaron: trabajo psicológico personal; medicina alternativa; practicar deporte; disminuir la carga laboral; realizar ejercicios de relajación; descansar; continuar con hobbies y actividades de ocio; cultivar el sentido del humor; fomentar la red de apoyo social y familiar; desahogarse; trabajar en equipo, y enfocarse en metas futuras. Algunos de los recursos mencionados coinciden con los utilizados por cuidadores formales en otros contextos culturales ${ }^{22}$.

La capacidad de desarrollar y mantener una actitud que aumente las "ganas de luchar (...), de aferrarte a la vida que te queda, disfrutar y vivir de eso" (Enfermera) apoya a la labor de los cuidadores y es vista como "una herramienta natural" (Voluntaria). Finalmente, frente a las cuestiones existenciales a las que se enfrentan, el cultivar su creencia religiosa y/o su espiritualidad fue otro factor importante. "Aquí hemos desarrollado no tanto una religión, sino una parte espiritual, y eso también ha sido una roca para apoyarme a mí porque sí he tenido ratos de angustia, tristeza, impotencia, no saber qué hacer" (Voluntaria). 
Al lado contrario del espectro se notó la falta de consciencia de la importancia del autocuidado y las excusas para no practicarlo: "No tengo mucho tiempo para cuidarme" (Médico) o "La fuerza del espíritu es tan grande que me muevo muchísimo y a veces me canso y no me doy cuenta" (Voluntaria). Como cuidar de sí mismos no se encuentra entre sus prioridades, en ocasiones no logran identificar sus propias necesidades y solo se preocupan cuando su salud física o emocional se ve afectada.

Satisfacción (44/84) y sentido (84/84). La satisfacción por el cuidado brindado estaba presente durante todo el proceso de cuidado hasta la muerte digna del paciente. "Me doy cuenta de que no se trata de salvar vidas... O sea, no es permitir que muera, sino que viva dignamente hasta el final...; eso sí es salvar vidas" (Voluntario). Simultáneamente, los entrevistados describieron que su labor y servicio humanizado y respetuoso tiene un sentido personal que les motivaba a continuar. "Son pacientes que necesitan mucho la parte afectiva, la parte emocional, no solo la parte física. El poder aportar en eso es una enorme satisfacción (...). El poder dar apoyo cuando ellos creen que ya no se puede hacer absolutamente nada y nosotros decir 'sí podemos todavía hacer cosas', dar todavía una opción (...), dar calidad de vida" (Psicóloga).

Esta capacidad de encontrar sentido y gozo en su labor es notable, puesto que se ha comprobado que la satisfacción tiene el potencial de disminuir la fatiga por compasión ${ }^{22,23}$, y se correlaciona positivamente con el cuidado emocional y espiritual, y con tener un equilibrio entre la vida personal y laboral24.

Barreras estatales (77/84). Las circunstancias externas relacionadas con la política pública y la carencia de provisión de servicios por parte del Estado fue la barrera más mencionada. Aquí se agruparon la falta de oportunidades de educación e investigación en cuidados paliativos; los obstáculos para registrar títulos y especialidades sanitarias obtenidas en el extranjero; la dificultad de acceso a medicamentos, y la inexistencia de apoyos sociales para pacientes y sus familiares. Los participantes no sienten apoyo por parte de las instituciones y las políticas de Estado. "No se tienen las herramientas suficientes para poder hacer cuidados paliativos a un nivel adecuado (...). A nivel del Ministerio de Salud no lo entienden, no entienden los tiempos que uno debe tener con los pacientes, los tiempos y los espacios para hablar con la familia" (Médico).

Estas barreras son las que generaban mayor frustración porque los cuidadores se perciben impotentes frente a procesos burocráticos y administrativos que afectan a los pacientes y en los cuales ellos no tienen injerencia. "El sistema nacional de salud no contempla lo que es un paciente con cáncer; no entiende. Cuando piensan en cáncer, piensan en quimioterapia, radioterapia y paliativos... ahora; antes, incluso sin paliativos. Es un sistema mucho más amplio, es nutrición, es trabajo social... Por ejemplo, ¿qué te haces con la mamá de tres hijos que deja tres hijos en la orfandad? O sea, son cosas que van más allá de la visión cuadrada que pueden tener ciertas autoridades con respecto a las enfermedades catastróficas. Sí creo que esa es una barrera que es muy triste, porque dices “cómo le ingreso si solo le quiero ingresar porque los hijos son muy chiquitos y no van a poder ayudarle a la mamá en tal o cual cosa?’ Debería haber cómo" (Médico).

\section{Discusión}

Las entrevistas revelaron factores potencialmente modificables para mejorar la calidad de vida profesional de los cuidadores. Los temas identificados se ubicaron en un continuum más que una categoría concreta, ya que las barreras fueron percibidas también como recursos cuando eran gestionadas adecuadamente. Mucho de lo identificado coincide con otras investigaciones, como la dificultad emocional en los cuidados paliativos pediátricos ${ }^{16}$, la necesidad de educación e inclusión de la enseñanza de cuidados paliativos ${ }^{21}$, el trabajo en equipo, la importancia del autocuidado ${ }^{22}$, los problemas asociados a los servicios de salud formal ${ }^{3}$, las dificultades de acceso a recursos y el equilibrar la vida personal y familiar ${ }^{25}$.

Conocer profundamente esta realidad ayuda a desarrollar intervenciones específicas para promover las aptitudes y demás factores identificados por los cuidadores como recursos para realizar su labor. Por ejemplo, fomentar ambientes laborales seguros con trabajo colaborativo y contar con espacios de aprendizaje y práctica para que los cuidadores tengan oportunidades de reflexionar y aprender de sus colegas $^{26}$. Igualmente, los resultados ayudan a expandir la comprensión de la realidad de los cuidadores formales de personas en cuidados paliativos, y también pueden ser de utilidad para empezar a explorar las necesidades de cuidadores en otras áreas.

Esta investigación concuerda con otras en las que el hecho de vivir el proceso de enfermedad y muerte de los pacientes puede despertar duelos personales en los cuidadores forma$l^{2} s^{27}$, y con el hecho de que los problemas con la administración del sistema de salud y la dedicación de su tiempo en superar barreras administrativas, en lugar de dedicarse a los pacientes y sus familias como ellos quisieran ${ }^{11}$, limita su labor. Ambos factores pueden desencadenar consecuencias emocionales negativas e incrementan el riesgo de burnout y de fatiga por compasión ${ }^{21}$. Como medida de prevención, las instituciones pueden facilitar talleres de manejo de emociones para gestionar este tipo de vivencias personales y sufrimientos. Asimismo, dada la complejidad del manejo pediátrico, se deben ofrecer espacios educativos específicos frente a situaciones de cronicidad y terminalidad en niños y adolescentes.

Mundialmente, la necesidad de cuidados paliativos ha sido ignorada como una prioridad dentro de los sistemas de salud porque las medidas de salud se enfocan en extender la vida y la productividad ${ }^{3}$. En el contexto específico de esta investigación, los participantes percibieron la falta de apoyo y de políticas estatales como una de las mayores frustraciones o barreras en el momento de realizar su trabajo. Esto revela la realidad del país, que no tiene unidades registradas de enfermedades crónicas ni políticas para su manejo y prevención ${ }^{28}$, existe limitación en la disponibilidad de morfina y posee pocos servicios de cuidados paliativos, los mismos que tienen un alcance desigual sin respaldos adecuados y con fondos basados principalmente en donaciones ${ }^{1}$.

\section{Conclusiones}

Este estudio muestra la potencialidad de convertir barreras en recursos para repercutir positivamente en el cuidador 
formal y en la calidad de su cuidado. Al igual que un estudio previo $^{22}$, se señala que la satisfacción y el sentido encontrado en el cuidar son fuentes de protección frente a las posibles barreras que enfrentan los cuidadores formales. Sin embargo, los resultados también destacan que, a nivel personal, se debe mejorar la gestión de las vivencias personales y la práctica de autocuidado; mientras que, a nivel social y estatal, es necesario aumentar el conocimiento y comprensión de la naturaleza de los cuidados paliativos y promover su enseñanza en todos los niveles de educación formal en las profesiones asociadas a los servicios de salud. Finalmente, se destaca la llamada al desarrollo e implementación efectiva de políticas estatales que garanticen las prestaciones médicas y sociales necesarias para servir a personas con enfermedades crónicas, degenerativas y en cuidados paliativos.

\section{Limitaciones y futuras líneas de investigación}

Este estudio contó con la participación de cuidadores formales de diversas disciplinas y de instituciones de varios campos de atención para apoyar a personas con enfermedades crónicas, degenerativas y en cuidados paliativos. Esto refleja la multidisciplinariedad que se requiere para el cuidado de personas en estas circunstancias. Empero, se deben tomar en cuenta ciertas limitaciones en el momento de interpretar los resultados. Únicamente participaron cuidadores formales de instituciones privadas; puede que los cuidadores que colaboren con instituciones públicas y atiendan a otro tipo de población presenten otros desafíos y recursos. Finalmente, se debe tomar en cuenta el contexto cultural y social de los participantes, que pertenecen a Quito, Ecuador. Para futuras investigaciones se sugiere combinar con medidas cuantitativas y ampliar la muestra para explorar la realidad de cuidadores formales en otros contextos laborales y culturales.

\section{Financiación}

Este estudio no recibió ningún tipo de apoyo económico de ninguna agencia pública, comercial o sin ánimo de lucro.

\section{Conflictos de intereses}

Las autoras de este artículo declaran no tener conflictos de intereses.

\section{Agradecimientos}

Agradecemos profundamente a las instituciones y a todos los cuidadores que participaron en el estudio por su apertura y colaboración.

\section{Ética y consentimiento informado}

El estudio fue aprobado por el Comité de Ética de la Universidad de Deusto con fecha 6 de octubre de 2016 (Ref.: ETK 3/16-17).

\section{Datos}

El guion completo de la entrevista puede ser solicitado contactando con Paula Hidalgo Andrade: paulahidalgo@ opendeusto.es.

\section{Bibliografía}

1. Organización Mundial de la Salud, Worldwide Palliative Care Alliance. Primer atlas mundial de las necesidades de cuidados paliativos no atendidas; 2014 [consultado el 11 de noviembre de 2015]. Disponible en: http://www.who.int/mediacentre/ news/releases/2014/palliative-care-20140128/es/

2. Family Caregiver Alliance. Definitions. 2014. [el 1 de noviembre de 2015]. Disponible en: https://www.caregiver.org/definitions-0

3. Knaul F, Farmer P, Krakauer E, De Lima L, Bhadelia A, Jiang Kwete $\mathrm{X}$, et al. Alleviating the access abyss in palliative care and pain relief - an imperative of universal health coverage: the Lancet Commission report. Lancet. 2018;391:1391-454.

4. Pérez-Godoy E, Moreno-Guerín A. Duelo y muerte en cuidadores familiares y profesionales de enfermos de Alzheimer. Gerokomos. 2007;18:68-71.

5. Faílde J, Carballo J, Lameiras M, Rodríguez Castro Y. Personalidad resistente, calidad de vida y burnout en profesionales de la salud de centros gerontológicos. Salud i Ciencia. 2013;19:599-606.

6. Figley C. Compassion fatigue: Psychotherapists' chronic lack of self-care. J Clin Psychol. 2002;58:1433-41.

7. Benito E, Arranz Carrillo de Albornoz P, Cansio López H. Herramientas para el autocuidado del profesional que atiende a personas que sufren. FMC - Medicine Formación Médica Continuada en Atención Primaria. 2011;18:59-65.

8. Astudillo W, Salinas A. Bases para mejorar la intervención de los cuidadores en cuidados paliativos. Notas Paliativas. 2011;12(1).

9. Atukunda R, Memiah P, Sibongile C. Care for the caregiver: stress relief and burnout among health workers in HIV care. GJ Med PH. 2013;2:1-7.

10. Payne N. Occupational stressors and coping as determinants of burnout in female hospice nurses. J Adv Nurs. 2001;33:396-405.

11. Keidel G. Burnout and compassion fatigue among hospice caregivers. Am J Hosp Palliat Care. 2002;19:200-5.

12. Van Mol M, Kompanje E, Benoit D, Bakker J, Nijkamp M. The Prevalence of compassion fatigue and burnout among healthcare professionals in Intensive Care Units: A systematic review. Plos One. 2015;10:e0136955.

13. Stamm BH. The Concise ProQOL Manual. Proqol.org. [Internet]. 2010 [consultado el 14 de 2015 Noviembre de 2015]. Disponible en: http://www.proqol.org/uploads/ProQOL_Concise_2ndEd_ 12-2010.pdf

14. Hernández-Vargas C, Llorens-Gumbau S, Rodríguez-Sánchez A. Empleados saludables y calidad de servicio en el sector sanitario: un estudio de caso. Anal Psicol. 2014;30:247-58.

15. Cohen-Katz J, Wiley S, Capuano T, Baker D, Shapiro S. The effects of mindfulness-based stress reduction on nurse stress and burnout, Part III. Holist Nurs Pract. 2005;19:78-86.

16. Lingard L, Kennedy TJ. Qualitative research in medical education. En: Swanwick T, editor. Understanding medical education: evidence, theory and practice. Oxford, UK: Wiley-Blackwell; 2010. p. 323-35.

17. Taylor S, Bogdan R. Introducción a los métodos cualitativos de investigación. Barcelona: Paidós; 2000.

18. O’Brien BC, Harris IB, Beckman TJ, Reed DA, Cook D. Standards for reporting qualitative research: a synthesis of recommendations. Acad Med. 2014;89:1245-51.

19. Guba E, Lincoln Y. Fourth generation evaluation. Newbury Park: Sage; 1989. 
20. St-Laurent-Gagnon T, Carnevale FA. Pediatric palliative care: a qualitative study of physician's perspectives in a tertiary care university hospital. J Palliat Care. 2008;24:26-30.

21. Williams-Reade J, Lamson AL, Knight SM, White MB, Ballard SM, Desai PP. Paediatric palliative care: a review of needs, obstacles and the future. J Nurs Manag. 2015;23:4-14.

22. Mota Vargas R, Mahtani-Chugani V, Solano Pallero M, Rivero Jiménez B, Cabo Domínguez R, Robles Alonso V. The transformation process for palliative care professionals: The metamorphosis, a qualitative research study. Palliat Med. 2015; 30:161-70.

23. Kelly L, Runge J, Spencer C. Predictors of compassion fatigue and compassion satisfaction in acute care nurses. J Nurs Scholarsh. 2015;47:522-8.
24. Alkema K, Linton J, Davies R. A study of the relationship between self-care, compassion satisfaction, compassion fatigue, and burnout among hospice professionals. J Soc Work End Life Palliat Care. 2008;4:101-19.

25. Nolte A, Downing C, Temane A, Hastings-Tolsma M. Compassion fatigue in nurses: A metasynthesis. J Clin Nurs. 2017;26:4364-78.

26. McAllister M, McKinnon J. The importance of teaching and learning resilience in the health disciplines: a critical review of the literature. Nurse Educ Today. 2009;29:371-9.

27. Durall M. Care of the caretaker: managing the grief process of health care professionals. Pediatr Ann. 2011;49:266-73.

28. Organización Mundial de la Salud. Enfermedades no transmisibles: perfiles de países, 2014. Disponible en: http://www.who. $\mathrm{int} / \mathrm{nmh} /$ countries/ecu_es.pdf?ua=1. 